\title{
Emotion Drives Attention: Detecting the Snake in the Grass
}

\author{
Arne Öhman, Anders Flykt, and Francisco Esteves \\ Karolinska Institute
}

\begin{abstract}
Participants searched for discrepant fear-relevant pictures (snakes or spiders) in grid-pattern arrays of fear-irrelevant pictures belonging to the same category (flowers or mushrooms) and vice versa. Fearrelevant pictures were found more quickly than fear-irrelevant ones. Fear-relevant, but not fear-irrelevant, search was unaffected by the location of the target in the display and by the number of distractors, which suggests parallel search for fear-relevant targets and serial search for fear-irrelevant targets. Participants specifically fearful of snakes but not spiders (or vice versa) showed facilitated search for the feared objects but did not differ from controls in search for nonfeared fear-relevant or fear-irrelevant, targets. Thus, evolutionary relevant threatening stimuli were effective in capturing attention, and this effect was further facilitated if the stimulus was emotionally provocative.
\end{abstract}

Mammals evolved in environments where resources and dangers were unpredictably distributed in space and time. The reproductive potential of individuals, therefore, was predicated on the ability to efficiently locate critically important events in the surroundings. Resources such as food and mating partners were the objects of active foraging, whereas dangers had to be reflexively detected to be adaptively avoided. Framed in this way, an important component of the adaptive problem concerns different varieties of selective attention. Following James (1890), researchers have commonly distinguished between active and passive attention. The former is conceptualized as goal-driven and voluntarily controlled in a top-down fashion, whereas the latter is stimulusdriven and governed by bottom-up perceptual processes. Thus, in foraging for food, mammals would rely on active, goal-driven processes, and in detecting threat, on passive, stimulus-driven attention. Indeed, James (1890, pp. 416-417) included threatening events such as "wild animals," "metallic things," "blows," and "blood" among stimuli likely automatically and reflexively to capture attention. In agreement with this distinction, there are experimental data suggesting a contrast between voluntary, effortdemanding attentional processes with a slow time course, and quickly dissipating selective processes that are rapidly and automatically activated by peripheral stimulus events (e.g., Jonides, 1981; Müller \& Rabbitt, 1989).

Arne Öhman, Anders Flykt, and Francisco Esteves, Department of Clinical Neuroscience, Karolinska Institute, Stockholm, Sweden.

Anders Flykt is now at the Department of Psychology, University of Geneva, Geneva, Switzerland. Francisco Esteves is now at the Department of Psychology, Universidade de Lusófona de Humanidades e Tecnologias, Lisbon, Portugal.

The research reported in this article was supported by grants from the Swedish Council for Research in the Humanities and Social Sciences. Experiments 1 and 2 were run at the Department of Clinical Psychology, University of Uppsala, Uppsala, Sweden. We are indebted to Erik Spangenberg and Erika Viklund for help in running Experiment 1.

Correspondence concerning this article should be addressed to Arne Öhman, Section of Psychology, Department of Clinical Neuroscience, Karolinska Institute and Hospital, Z6, S-171 76 Stockholm, Sweden. Electronic mail may be sent to arne.ohman@cns.ki.se.
To detect threatening events outside the spotlight of focused, conscious attention, there must be perceptual processes that automatically scan and analyze the perceptual field. Most investigators appear to agree that there is an important distinction between preattentive and postattentive visual attention. The former is fast, automatic, and parallel and works on low-level stimulus features with the primary objective of delineating objects in the spatial surrounding. The latter is slow, deliberate, and serial and is concerned with more complex inferential and interpretative processes in identifying the located objects in perceptual awareness (e.g., Johnston \& Dark, 1986; Julesz \& Bergen, 1983; Posner, 1978; Schneider, Dumais, \& Shiffrin, 1984; Treisman, 1988). When unexpectedly encountered, peripheral significant events may interrupt ongoing processing and call for prioritized postattentive processing (Öhman, 1979). This stimulus-driven call for processing resources is associated with phasic psychophysiological activation manifested as orienting responses, which facilitates further sensory processing of the stimulus (Graham, 1992; Sokolov, 1963).

In this perspective, evolutionarily relevant threats may be "tagged" to get priority for processing, much like other types of critical events, such as sudden visual onsets, which appear to automatically guide attention to the appropriate region of visual space (Posner, 1980; Yantis \& Johnson, 1990). However, peripheral stimuli do not work in isolation to capture attention but typically interact with more or less explicit goal-driven attentional processes (Yantis, 1998). Folk, Remington, and Johnston (1992) demonstrated that there are attention control settings that tune the likelihood of stimuli to capture attention in accordance with their relevance for the current goals of the individual. Stimuli that are inconsistent with such attention control settings, on the other hand, are more easily ignored.

Because of survival relevance, there might be sets of stimulus features that capture attention because of control settings "by default" (Folk et al., 1992); that is, they may become the target of attention even in the absence or in spite of an explicit attention control setting. Thus, stimuli related to recurrent survival threats in the environment of evolutionary adaptedness (Tooby \& Cosmides, 1990) may have been selected by evolution to become more or less automatic triggers of attention. Other sets of effective attention triggers may reflect ontogenetic contingencies in the sense that 
their attentional potency derives from the learning history, or the present state (e.g., the level of anxiety; see, e.g., Mogg \& Bradley, 1998), of the individual.

From these considerations one would expect that threat stimuli owing their fear-relevance to evolutionary contingencies, such as snakes, spiders, and angry faces (see Öhman \& Mineka, 2001), would be likely to capture attention quite automatically. Indeed, some of us are familiar with the experience of the seemingly automatic focusing of attention on the snake that is resting in the grass a few steps ahead along the paths we are treading. Sometimes one may even freeze a fraction of a second later to realize that the "snake" was merely a twig. Furthermore, it is often the spider phobic individual in the company who discovers the spider inconspicuously moving across the ceiling.

Automatic activation of fear in these types of situations was incorporated into a model of fear activation proposed by Öhman (1993, 2000). According to this model, there is a system that automatically evaluates the significance of the output from a feature detection perceptual system. Evolutionarily derived threatening features get preferential access to this system, as well as to an arousal system, and therefore they are likely to shape the further processing of the stimuli, of which they are parts, up to an eventual, consciously perceived threat. This model was based on a series of studies demonstrating psychophysiological responses to fear stimuli (snakes, spiders, and angry faces) that because of backward masking were blocked from conscious processing (see Dimberg \& Öhman, 1996, and Öhman \& Mineka, 2001, for reviews). However, even though these results document preferential automatic processing of evolutionarily fear-relevant stimuli, they do not address the issue of preattentive selection of significant stimuli because the participants were exposed to only one stimulus at a time.

To examine automatic capture of attention by fear-relevant stimuli, Hansen and Hansen (1988) exposed research participants to complex matrices of visual stimuli with the task of pressing different buttons depending on whether all stimuli in a matrix were similar or whether it included a discrepant stimulus. They reported faster detection of a deviant threatening angry face in a background crowd of happy faces than vice versa. This anger superiority effect, furthermore, was reported to be unaffected by the size of the background crowd. Thus, detection of fear-relevant targets occurred as a "pop-out" effect of preattentive origin (see, e.g., Treisman, 1988). In agreement with the evolutionary scenario, as well as with Öhman's (1993) model, this interpretation suggested that evolutionarily significant threat stimuli were automatically detected in a complex visual display.

However, there are several problems with this interpretation (see Hansen \& Hansen, 1994). Most important, an error of experimental design confounded Hansen and Hansen's (1988) report of a pop-out effect. In the most critical experiment, only two angry and happy faces were used, and both the angry faces had characteristic dark patches, which provided unique low-level physical features, apparently accounting for the pop-out effect (Purcell, Stewart, \& Skov, 1996). Thus, the original Hansen and Hansen (1988) data cannot be invoked as support for automatic selection of threatening stimuli. Öhman, Lundqvist, and Esteves (2001), however, confirmed faster detection of angry than of happy (or sad) faces among neutral as well as emotional distractor faces, using wellcontrolled schematic facial stimuli in which identical physical features were used differentially to generate different emotional facial expressions. Furthermore, there is good evidence to support that angry, but not happy, faces can activate psychophysiological responses even though presented masked by neutral faces (Esteves, Dimberg, \& Öhman, 1994; Parra, Esteves, Flykt, \& Öhman, 1997; see review by Dimberg \& Öhman, 1996). Thus, for one class of stimuli whose threat potential is likely to derive from evolutionary contingencies, angry faces (e.g., Öhman \& Dimberg, 1984; Öhman et al., 2001), there is evidence both of automatic emotional response activation (e.g., Esteves et al., 1994) and of efficient capture of attention (Öhman et al., 2001).

Because it has been demonstrated that small animal stimuli can control psychophysiological responses from a mere preattentive level of information processing (e.g., Öhman, 1993; Öhman \& Mineka, 2001), it is interesting to examine whether the coupling of automatic emotional activation and efficient capture of attention that holds for angry faces also is true for another class of evolutionary fear-relevant stimuli, snakes and spiders. Thus, the purpose of the present series of experiments was to determine whether the facility of processing snake and spider stimuli at an automatic level also includes efficient selection of such stimuli from complex visual displays. Inspired by the methodology pioneered by Hansen and Hansen (1988), we exposed our research participants to two fear-relevant (snakes and spiders) and two fear-irrelevant (flowers and mushrooms) categories of stimuli. The task of the participants was to detect discrepant stimuli in matrices of pictures, either composed only of exemplars from the same category (e.g., flowers) or including a single target exemplar from a different category (e.g., a snake) among those of the background category (e.g., flowers). Our hypothesis was that participants would be faster to detect fear-relevant discrepant stimuli among fear-irrelevant visual stimuli than vice versa and that this effect should be independent of the number of distractors. We also modeled animal phobic individuals' seemingly preferential discovery of the feared animal in the environment by examining whether the effect was modulated by the emotional impact of the stimuli. This was achieved by comparing the performance of participants fearful of snakes or spiders with nonfearful participants. The underlying assumption here was that intense fear of a category of stimuli would modulate the attention control setting (Folk et al., 1992) to make stimuli from this category more salient in automatically grabbing attention.

\section{Experiment 1}

The purpose of this experiment was to test whether research participants would be faster in discovering a discrepant fearrelevant stimulus against a background of fear-irrelevant stimuli than the other way round. The participants were exposed to complex visual stimuli composed of nine individual pictures arranged in $3 \times 3$ matrices. They were asked to respond by pressing different keys depending on whether all pictures came from the same category (snakes, spiders, flowers, or mushrooms) or whether there was a discrepant stimulus in the matrix. Given the evolutionary significance of snakes and spiders as potential survival threats (e.g., Öhman, Dimberg, \& Öst, 1985; Öhman \& Mineka, 2001), we hypothesized that the participants would be faster in finding pictures of snakes and spiders against a back- 
ground of flowers and mushrooms, than flowers and mushrooms against a background of snakes and spiders.

\section{Method}

Participants. Twenty-five psychology students, 12 men and 13 women, volunteered on an informed consent basis to perform as research participants to gain course credits. They were not screened for fear of snakes or spiders. Their age ranged from 21 to 41 years with a mean of 28 years.

Apparatus. The participant was seated in a sound-attenuating chamber in front of a milk glass screen upon which the stimuli were back projected from a projector outside the chamber. Color slide stimuli were presented from a Rotomatic projector equipped with shutters (Vincent \& Associates, Rochester, New York) to accurately control exposure duration. Reaction times (RTs) were produced by microswitches, one held in each hand, and were measured to the nearest millisecond by electronic counters that were started when the shutter opened and were stopped by the response.

Stimuli. Four different categories of color slides (snakes, spiders, mushrooms, and flowers), each containing nine individual exemplars, were used to construct the stimulus matrices. A stimulus matrix (three rows and three columns) consisted of either nine different pictures from the same category (distractors) or eight different distractors from the same category and a discrepant target picture. The discrepant picture belonged to another category and was placed in one of the nine possible positions, with position of the target systematically balanced over the possible positions across trials. Fear-relevant stimuli (snakes and spiders) were targets in matrices with fear-irrelevant stimuli (flowers or mushrooms) as distractors and vice versa. A fear-relevant picture could never serve as a target among fearrelevant distractors, nor could a fear-irrelevant picture be a target among fear-irrelevant distractors. In other words, a flower matrix as well as a mushroom matrix could contain a spider or a snake target, and a spider or a snake matrix could contain a flower or a mushroom target. This yields eight different combinations, snake among flowers, spider among flowers, snake among mushrooms, and so on. The ninth picture, the one that did not match the others for category, could be presented in any one of the nine different positions. This results in $72(8 \times 9)$ stimuli with a target. To balance the design, we constructed another 72 stimuli without target picture, which made a total of 144 stimuli. There were nine different configurations with regard to specific stimuli in specific locations of the matrices for each category, with no pictures appearing in the same position more than once. The configuration of distractors was different the two times that a discrepant category was presented in the same location. For example, if the distractors were flowers they were organized in different ways when a snake or a spider served as the target. In this way the configuration of individual pictures in the matrices could not be used as clues for targets at a certain position.

The presentation order of the matrices was not blocked by condition but was randomized with the following restrictions: no more than three matrices with or without a target were presented in sequence, and two matrices with a target in the same position were never presented after each other.

Procedure. The size of the matrix projected at eye level on the screen $1 \mathrm{~m}$ in front of the participant was $20 \mathrm{~cm}$ high and $30 \mathrm{~cm}$ wide (approximately $11.5^{\circ} \times 16.5^{\circ}$ in visual angles). Matrices were exposed for 1,200 $\mathrm{ms}$ and were heralded by the appearance of a red fixation spot, which was projected at the center of the screen for $1 \mathrm{~s}$ before presentation of each matrix. The participants were instructed to fixate the red spot and to respond as fast as possible with either of the response buttons held in each hand. The task was described as involving the detection of targets among distractors, with the targets defined as pictures whose object came from a discrepant category from that of the object depicted in all the distractors. Participants were told that if all the pictures in a display belonged to the same category, they should press the microswitch held in the nonpreferred (typically left) hand, and if there was a target in the display, they should press the microswitch in the preferred (typically right) hand. The micro- switch indicating that there was a target in the matrix was always held in the preferred hand (one participant was left handed), because the most important question of the experiment pertained to differences in response latencies for identifying target stimuli. The participants were explicitly told that half of the displays contained a target and that they should try to be as fast and as accurate as possible in their responses. The time between successive stimulus exposures was decided by the participants, who indicated when they were ready for the next exposure by saying "next."

Each session started with 11 practice trials, which included stimuli both with and without targets. None of the matrices used in the practice trials appeared among the 144 matrices used in the main experiment. Eight different stimuli orders were used in the experiment.

Statistical analyses. Only RTs to correctly identified targets and correctly rejected nontarget matrices were included in the analyses. RTs for errors trials were replaced by the mean for the particular target distractor combination for matrices with targets, and by the mean for the particular distractor condition for matrices without targets. Separate analyses of variance were performed for detection latencies to matrices with and without targets. To reduce the effect of outliers and to normalize the distributions (Ratcliff, 1993), we inverted reaction time data (1,000/RT in ms) before reduction and analyses. ${ }^{1}$ However, to facilitate understanding of the data, the figures and means are presented as RTs in ms. RTs to targets were analyzed in a $2 \times 3 \times 3$ within-subject design with fear relevance of the target, and rows and columns in the matrices as independent variables. Matrices without targets were analyzed with fear relevance of the distractors as the independent variable. The Greenhouse-Geiser $\epsilon$ was used to correct the degrees of freedom for variables with more than two levels. Errors were analysed in a $2 \times 2$ analysis of variance incorporating target versus no target and fear relevance of the distractors as factors. Tukey tests were used for follow-up analyses.

\section{Results}

The mean RTs to fear-relevant and fear-irrelevant targets for all positions in the matrix are displayed in Figure 1. In agreement with the main hypothesis of the study, the analysis showed that the participants were clearly faster in detecting fear-relevant than fear-irrelevant targets, $F(1,24)=22.29, p<.0001$.

The overall effect of row, $F(2,48)=11.12, p<.0001, \epsilon=.88$, showed that the participants were faster to detect targets in the middle as compared with the upper $(p<.01)$ and lower $(p<.05)$ rows. According to the significant interaction between fear relevance and rows, $F(2,48)=4.93, p<.02, \epsilon=.94$, the effect of rows was more obvious for fear-irrelevant than for fear-relevant targets. Whereas mean RTs to fear-relevant matrices were similar across rows, the RTs to fear-irrelevant targets were faster to the middle than to the upper and lower rows. As a result, the detection of fear-relevant targets was faster than that of fear-irrelevant targets for more peripheral targets (i.e., in the upper and lower rows; $p<.05$ for both), whereas fear relevance had no effect in the middle row.

The interactions between rows and columns and between fear relevance, rows, and columns, $F(4,96)=3.41, p<.02, \epsilon=.85$, and $F(4,96)=7.92, p<.0001, \epsilon=.60$, respectively, provided further evidence for different search patterns for fear-irrelevant and fear-relevant targets. With fear-irrelevant targets, participants appeared to search first the middle row from left to right, then the

\footnotetext{
1 The results were substantially the same when the analyses were performed on RTs filtered for outliers (three standard deviations above or below the means).
} 


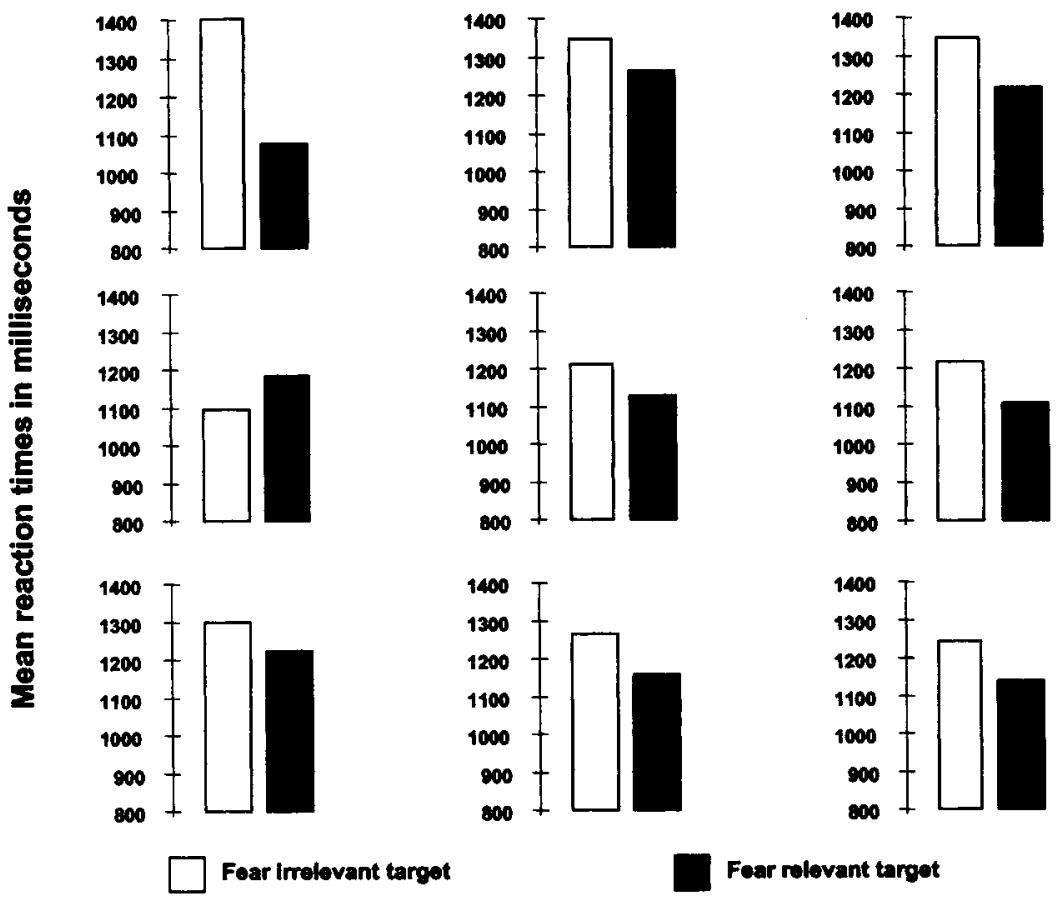

Figure 1. Mean reaction time to locate a discrepant fear-relevant (snake or spider) or fear-irrelevant (flower or mushroom) target stimulus among fear-irrelevant or fear-relevant distractors, respectively, for each position in the $3 \times 3$ matrices in Experiment 1 .

lower row from right to left, and finally the upper row from right to left. As a result, whereas there were no differences between rows in the middle and right columns, for the left column the participants were clearly faster in detecting the middle than the lower and upper cells ( $p<.01$ for both). With fear-relevant targets, on the other hand, there were no significant differences between rows in any of the columns, suggesting that the location of the target in the matrix was less important in this condition.

There were no differences in latencies to decide that targets were not present as a function of fear relevance. The error rates were low $(<15 \%)$ and did not differ as a function of fear relevance for either the targets or the distractors.

\section{Discussion}

In support of the main hypothesis for the experiment, participants proved faster in detecting fear-relevant targets among fearirrelevant distractors than vice versa. Thus, snakes and spiders against flowers or mushrooms resulted in shorter detection latencies than flowers and mushrooms against snakes or spiders. These differences could not be straightforwardly attributed to differential ease of processing the two types of stimuli, because the latency to decide that a target was not present in a matrix was independent of the fear relevance of the distractors. This conclusion is further vindicated by the lack of effect of fear relevance on error rates.

Interestingly, the position of the target in the $3 \times 3$ matrix of pictures appeared to be more important for fear-irrelevant than for fear-relevant targets. Thus, whereas a systematic search pattern appeared to be present in the ordering of RTs according to positions for fear-irrelevant targets, there were no systematic effects of position for fear-relevant targets. Indeed, because the effect of fear relevance was significant only at the upper and lower rows, the advantage of fear-relevant targets was apparent only when attention had to be shifted from the middle row that was initially fixated. For the stimuli presented in the position immediately to the left of the center fixation point, and thus initially analyzed by the right cerebral hemisphere, there was even a tendency for faster RTs to fear-irrelevant targets. For all other positions, however, and particularly for those above or below fixation, for which shifts of attention were required, the detection latencies were shorter for fear-relevant targets. It appears, therefore, that fear-relevant targets prompted more rapid shifts of attention from one picture to another. Thus, fear relevant targets may have been automatically and preattentively detected in a pop-out process (e.g., Hanson \& Hanson, 1988; Treisman \& Gelade, 1980), independently of their spatial location in the matrix. However, this conclusion must remain tentative for at least two reasons. First, it is based on mean RTs over all participants. This does not necessarily imply that the pattern was discernible in any individual participant. Second, the ordering effect may have been confounded by individual target pictures, because the particular pictures appearing as targets in particular positions was not completely counterbalanced.

To provide a stronger basis on which conclusions about efficiencies of search and a potential pop-out effect could be based, we needed to perform an experiment incorporating the principal manipulation to assess efficiency of search (Wolfe, 1998), that is, varying the number of distractor stimuli. Therefore, in Experiment 2 we examined detection latencies for fear-relevant and fear-irrelevant stimuli in large $(3 \times 3)$ and small $(2 \times 2)$ matrices. 


\section{Experiment 2}

\section{Method}

Participants. Thirty students volunteered to serve in the experiment, 15 women and 15 men. A majority of them were psychology students who gained course credit for their participation. The age range of the participants was 16 to 37 years, with mean age of 27 years.

Stimuli. We retained 128 stimuli from the previous experiment, excluding matrices with the deviant picture appearing in the center positions of the matrix and the corresponding matrices without targets. In addition to these matrices, new matrices with pictures only in the four corner positions of the $3 \times 3$ matrices were constructed to constitute the set of small, $2 \times 2$, matrices. Because they used only the corner positions, the small matrices occupied the same visual angle as the large ones. The space in between the pictures was black. The matrices for four pictures were constructed according to similar principles as used in the $3 \times 3$ matrices. There were 32 individual matrices with a target and 32 without. To balance the number of $2 \times 2$ matrices with that of the $3 \times 3$ matrices, we duplicated each of the 64 stimuli, yielding a grand total of 256 matrices.

Procedure. The experimental procedure was identical to that used in Experiment 1.

Statistical analysis. As in Experiment 1, data were analyzed separately for matrices with and without targets for inverted RTs. For the analysis based on latencies to detect targets, a $2 \times 2$, Fear Relevance $\times$ Matrix Size ANOVA was performed on data collapsed over positions in the matrices. To examine position effects, one analysis was performed in which the average (inverted) RT in the left and the right columns of the $2 \times 2$ and $3 \times 3$ matrices was included; another analysis was based on the average for upper and lower rows of the matrices. Matrices without targets were examined in a $2 \times 2$ Fear Relevance $\times$ Matrix Size ANOVA. Separate analyses for errors were performed for matrices with and without targets, using a similar design to those used for RT data.

\section{Results}

RT data for matrices that included a target are shown in Figure 2. In agreement with the results from Experiment 1, the participants were clearly faster in detecting fear-relevant than fear-irrelevant targets, $F(1,29)=38.46, p<.001$. Detection times were faster with the $2 \times 2$ than with the $3 \times 3$ matrix, $F(1$, $29)=42.79, p<.001$. However, the significant interaction between these two variables, $F(1,29)=9.92, p<.01$, indicated
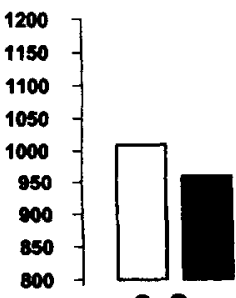

$2 \times 2$

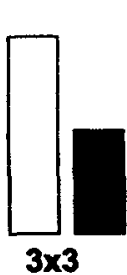

Fear irrelevant target Fear relevant target

Figure 2. Mean reaction time to locate a discrepant fear-relevant (snake or spider) or fear-irrelevant (flower or mushroom) target stimulus among fear-irrelevant or fear-relevant distractors, respectively, in small $(2 \times 2)$ and large $(3 \times 3)$ stimulus matrices in Experiment 2 .
Table 1

Reaction Time (ms) for Matrices Without Targets in Experiment 2

\begin{tabular}{ccc}
\hline Matrix size & Fear irrelevant & Fear relevant \\
\hline $2 \times 2$ & 1,111 & 1,046 \\
$3 \times 3$ & 1,158 & 1,159 \\
\hline
\end{tabular}

that the effect of matrix size differed as a function of fear relevance of the target. Indeed, as is clear in Figure 2, there was a strong effect of matrix size with fear-irrelevant targets, $p<.001$, whereas there was no effect of this variable for fear-relevant targets.

In the analysis examining the left-right position effects, both the two-way interactions between position and fear relevance, $F(1$, $29)=20.38, p<.001$, and position and matrix size, $F(1$, 29) $=10.87, p<.01$, as well as the three-way interaction between position, fear relevance, and matrix size, $F(1,29)=10.24, p<$ .01 , were significant. This pattern of findings could be attributed to the fact that RTs to fear-relevant targets were remarkably stable across left-right positions and matrix sizes (varying between 950 and $964 \mathrm{~ms}$ ), whereas the RTs to fear-irrelevant targets were faster with the small matrix and were modified depending on whether the target occurred in the left or the right column. Thus, whereas RTs to fear-irrelevant targets were faster with the target in the right column for the $2 \times 2$ matrix, the opposite was true with the $3 \times 3$ matrix. In the analysis performed on the means for the upper and lower rows, the two-way interactions between position and fear relevance, $F(1,29)=43.31, p<.0001$, and position and matrix size, $F(1,29)=8.67, p<.01$, were significant. The former effect was due to a larger effect of fear relevance for targets presented in the upper row, and the latter to a smaller difference between rows in the small than in the large matrix. Again, this pattern partly reflected less of an effect of position on RTs to fear-relevant than to fear-irrelevant targets.

For matrices without targets (Table 1), participants were considerably faster to decide that a target was not present if the matrix was small, $F(1,29)=104.59, p<.0001$. They were also overall faster to decide that a target was not present if the distractors were fear relevant than if they were fear irrelevant, $F(1,29)=7.17, p<$ .01 . However, according to the interaction between fear relevance and matrix size, $F(1,29)=9.95, p<.01$, the effect of fear relevance pertained exclusively to the $2 \times 2$ matrices $(p<.01)$.

Errors were fewer with fear-relevant than with fear-irrelevant targets, $F(1,29)=10.18, p<.001$, and with small matrices, $F(1$, $29)=30.65, p<.0001$, with no interaction between these two variables (see Table 2). For matrices without targets, there were more errors with the larger matrix and with fear-relevant distractors than in any of the other conditions, according to the interaction between matrix size and fear relevance, $F(1,29)=4.38, p<.05$ (see Table 2).

\section{Discussion}

In agreement with the results from Experiment 1, participants were significantly faster to find a discrepant stimulus in a matrix if it was fear relevant than if it was fear irrelevant. Furthermore, the latency to find fear-relevant stimuli was not significantly prolonged when the number of distractors was increased from three to 
Table 2

Proportion of Errors of Matrices With and Without Targets in Experiment 2

\begin{tabular}{ccccccc}
\hline & \multicolumn{2}{c}{ With targets } & & \multicolumn{2}{c}{$\begin{array}{c}\text { Without targets } \\
\text { (distractors) }\end{array}$} \\
\cline { 2 - 3 } \cline { 5 - 6 } Matrix size & $\begin{array}{c}\text { Fear } \\
\text { relevant }\end{array}$ & $\begin{array}{c}\text { Fear } \\
\text { irrelevant }\end{array}$ & & $\begin{array}{c}\text { Fear } \\
\text { relevant }\end{array}$ & $\begin{array}{c}\text { Fear } \\
\text { irrelevant }\end{array}$ \\
\cline { 1 - 3 } $2 \times 2$ & .038 & .060 & & .033 & .039 \\
$3 \times 3$ & .087 & .125 & & .053 & .031 \\
\hline
\end{tabular}

Note. "With targets" indicates the proportion of errors with fear-relevant and fear-irrelevant targets. "Without targets" indicates the proportion of errors for fear-relevant and fear-irrelevant distractors.

eight, that is, in the comparison between $2 \times 2$ and $3 \times 3$ matrices. In fact, even though there were five more pictures to scan in the larger matrix, the mean increase in RT to locate fear-relevant stimuli was only about $15 \mathrm{~ms}$. Thus, the increase was about 3 ms/added distractor, which is clearly within the limits of a maximum of the 5-6 ms/item that has been required for parallel searches in the literature (e.g., Treisman \& Souther, 1985). For fear-irrelevant targets, on the other hand, there was a highly significant increase in RT from the small to the large matrix (close to $20 \mathrm{~ms}$ increase/item), suggesting that the participants used more of a serial search to locate fear-irrelevant targets.

The effects of matrix size were congruent with the data generated in the analyses of target positions, which indicated that detection of fear-relevant targets was more independent of target position than was detection of fear-irrelevant targets. However, the same caveats as those expressed regarding the position data in Experiment 1 are valid here too, because target pictures were not completely counterbalanced across positions. Nevertheless, the data from Experiment 2 appear to provide strong support for the notion that fear-relevant targets are located through a parallel search, which stands in clear contrast to the serial process that appears to characterize the search for fear-irrelevant targets (e.g., Hansen \& Hansen, 1988; Treisman \& Gelade, 1980). However, some caution is called for in accepting this conclusion. First, it is debatable whether increase versus no increase in detection latency as a function of the number of searched items distinguishes between qualitatively different search processes or merely between more or less efficient searches (Wolfe, 1998). Second, Experiment 2 sampled a rather limited range of display sizes, and there is evidence that large displays (e.g., $>10$ items) may engage different attentional processes than small displays (Pashler, 1987). ${ }^{2}$ Third, even though schematic angry faces were more quickly detected than schematic happy faces regardless of display size, increased latency as a function of display size depended on the perceptual discriminability of targets and distractors rather than on the threat value of the target (Öhman et al., 2001). Thus, when the search concerned happy and angry targets against neutral distractors, there was little increase in detection latency for either target up to displays as large as 25 items. Similarly, with angry targets against happy distractors, or vice versa, there was a clear increase in detection latency with larger displays both for angry and happy targets. Thus, it must be remembered that perceptual characteristics such as target-distractor discriminability are a central determinant of search efficiency (see Wolfe, 1998).
The results from the analysis of matrices without targets showed a very strong effect of matrix size and faster decision that fearrelevant distractors did not include a discrepant target stimulus. As in Experiment 1, this effect was not evident in the $3 \times 3$ matrix, but it was so strong in the $2 \times 2$ matrix that it resulted not only in a reliable interaction but also in a significant main effect. In combination with the fact that there were fewer errors to fearrelevant pictures, these data suggest that participants actually were more efficient in processing the fear-relevant material. Thus, not only were the participants faster in shifting their attention to fear-relevant targets, as shown by the shorter detection latencies, but they also appeared faster in disengaging attention (see Posner \& Peterson, 1990) from fear-relevant stimuli, at least for small matrices, as shown by the shorter latency to decide that a discrepant target was not present in a uniform display of fear-relevant stimuli.

The results from Experiments 1 and 2 show quite clearly that people more or less automatically turn their attention to stimuli involving some degree of threat, at least if the threat is provided by potentially harmful small animals. Thus, it appears that humans have an attention control setting (Folk et al., 1992) to automatically attend to stimuli implying some degree of threat to their forefathers throughout evolution. However, it is clear that the stimuli we used do not pose threat in any real sense. For instance, it could be argued that their quick detection simply reflected that they belonged to a common (and potentially more interesting) category of (potentially moving) objects, animals, in contrast to the less interesting (and stationary) plants that constituted the fearirrelevant stimuli. Thus, the data from Experiments 1 and 2 could be accounted for by factors other than threat. Indeed, among the unselected participants used in the studies, it is likely that the responses to fear-relevant stimuli had minimal, if any, emotional intensity. An important question raised by our findings, therefore, is whether the recruitment of attention would be even more effective if the stimuli were actually perceived as dangerous and emotion provoking by the research participants. Or, framed as a question, would fearful participants show evidence of a more biased attention control setting than nonfearful participants? Persons suffering from phobias of small animals appear indeed to respond emotionally to pictures of their feared material as if they were dangerous (e.g., Globisch, Hamm, Esteves, \& Öhman, 1999; Hamm, Globisch, Cuthbert, \& Vaitl, 1997) and to rate the phobic object as more objectively dangerous than control participants (Thorpe \& Salkovskis, 1995). Thus, if participants were recruited who were highly fearful of snakes or spiders, would the bias toward discovering such creatures be further enhanced compared with what we have observed in nonfearful participants in the preceding two experiments? Another possibility, of course, is that fearful participants would show defensive avoidance and thus take longer to discover fear-relevant targets among fear-irrelevant distractors than vice versa. In Experiment 3, we attempted to answer this question by comparing detection of feared and nonfeared fear-relevant stimuli in participants selected to fear either snakes or

\footnotetext{
${ }^{2}$ The focus of this article, however, was on the contrast between target absent/target present decisions, rather on parallel versus serial search for targets, as in the present study.
} 
spiders, but not both. A nonfearful control group was also included.

\section{Experiment 3}

\section{Method}

Participants. About 130 students at Karolinska Institute (age range $=$ 20-38 years) answered true-false items about their fear of snakes and spiders in the snake (30 items) and spider (31 items) questionnaires (Klorman, Weerts, Hastings, Melamed, \& Lang, 1974). Students who scored high on either fear of snakes or fear of spiders, but not on both, as well as those scoring low on both, were recruited as research participants. The high-fear participants included 8 scoring high on the snake and 9 scoring high on the spider questionnaire. The cutoff score was 15 for women and 10 for men for both questionnaires, which roughly corresponded to the 90th percentile in the female and male distributions, respectively. To ensure that the fear was specific to snakes or spiders, high-fear participants had to score below the median on the other questionnaire (e.g., spider questionnaire for snake fearful participants). The goal of the selection procedure was to select high-fear groups whose level of fear was in the phobic range, but no effort was made to diagnose formally whether they fulfilled other criteria (e.g., debilitating avoidance behavior) of animal phobia. The 17 specifically fearful participants (14 women and $3 \mathrm{men}$ ) were compared with a control group of 17 low-fear participants (same gender distribution) scoring below the median on both questionnaires. All participants were paid for their participation.

Apparatus. We conducted stimulus presentation and timing as well as RT measurements using the Micro Electronic Laboratory (MEL) software (Schneider, 1988) and a PC computer, which controlled a Kodak Ektapro 7000 with internal high-speed shutters that presented the stimuli.

Stimuli. The same 256 stimuli as in Experiment 2 were used. Thus, matrix size and fear relevance were included as factors in the design. No more than 4 participants had the stimuli presented in the same order (balanced between groups).

Procedure. The procedure in this experiment was identical to that in Experiment 2 with two exceptions. First, the center of the screen was indicated by a small square of silver colored tape, on which the participants were instructed to fixate when asking for the next stimulus. Second, stimulus exposure was terminated by the button press.

Statistical analysis. The data were analyzed as in Experiment 2 with the addition of a between-subjects factor in the analysis representing high versus low fear. We examined the predicted faster reaction time to feared versus nonfeared fear-relevant targets in fearful participants by a planned contrast using a $t$ test.

\section{Results}

The results from matrices with targets (see Figure 3) were consistent with Experiments 1 and 2 with regard to the strong effect of fear relevance, $F(1,32)=120.82, p<.0001$, with faster detection of fear relevant targets. Similarly, they were consistent with Experiment 2 with regard to a reliable effect of matrix size, $F(1,32)=7.61, p<.01$, and the significant interaction between fear relevance and matrix size, $F(1,32)=24.60, p<.001$. Again this latter effect was due to the fact that matrix size had a clear effect for the fear irrelevant ( $p<.01$; lower panels of Figure 3 ) but not for the fear-relevant targets ( $p>.05$; upper panels).

There were no overall difference in RT to detect targets between fearful and nonfearful participants, $F(1,32)<1$, but the effect of fear relevance of the target was stronger for fearful than for nonfearful participants, $F(1,32)=10.23, p<.01$.

Confirming the main hypothesis of the experiment, fearful participants were faster to detect their feared than their nonfeared fear-relevant target, $t(16)=2.48, p<.02$ (for the means across matrix size, compare the two upper panels of Figure 3 ). The nonfearful participants, for whom this was a dummy variable, of course did not differentiate between these two stimulus categories. For both groups, however, detection latencies were faster for fear-relevant than for fear-irrelevant targets (compare the upper and lower panels of Figure 3). In combination, these effects resulted in a significant three-way interaction between groups, feared target, and fear relevance, $F(1,32)=6.44, p<.02$,
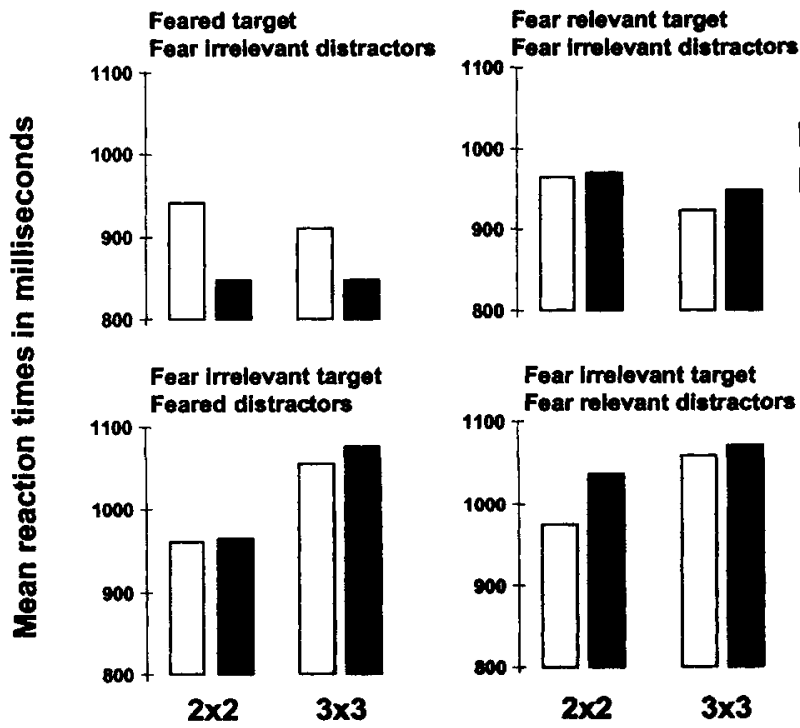

Figure 3. Mean reaction time for fearful and nonfearful participants to locate a discrepant fear-relevant (snake or spider) or fear-irrelevant (flower or mushroom) target stimulus among fear-irrelevant or fear-relevant distractors, respectively, in small $(2 \times 2)$ and large $(3 \times 3)$ stimulus matrices in Experiment 3 . 
confirming that fearful participants were faster than controls only with the feared target.

Matrices without targets (see Figure 4) were analyzed in a $2 \times$ $2 \times 3$ (Group $\times$ Matrix Size $\times$ Picture Type: feared distractors, nonfeared fear-relevant distractors, fear-irrelevant distractors) ANOVA. It took significantly longer to decide that a target was not present among the distractors in the large matrices, $F(1$, $32)=80.52, p<.0001$, and in matrices with fear-irrelevant as opposed to fear-relevant and feared distractors, $F(2,64)=13.25$, $p<.001$ and Tukey tests $(p<.01)$. This effect was somewhat clearer with the large than with the small matrix, $F(2,64)=3.84$, $p<.03$, for the interaction between matrix size and type of stimulus.

The analysis of errors for matrices with targets showed overall more errors in large than in small matrices, $F(1,32)=11.42, p<$ .002 , and among fearful as opposed to nonfearful participants, $F(1$, $32)=5.34, p<.03$. However, according to the interaction between fearfulness and fear relevance of the distractors, $F(1$, $32)=5.68, p<.02$, the fearful participants made more errors than low-fear participants only with fear-irrelevant targets. With fearrelevant targets they performed as well as, or even better (particularly with feared targets) than nonfearful participants (see Table 3). Given that matrices with fear-irrelevant targets had fearrelevant distractors, this implies that high-fear participants were distracted to miss targets when exposed to fear-relevant distractors. As shown in Table 3, the highest error rate occurred for fearful participants with feared distractors in large matrices. For matrices without targets (right part of Table 3), the error rates were low and did not differ between groups or conditions.

\section{Discussion}

The results from Experiment 3 fully confirmed the findings in the two previous experiments with regard to target fear relevance and matrix size. Thus, overall the participants were faster in finding snakes and spiders against backgrounds of flowers and mushrooms than the other way round. Furthermore, whereas it took longer to locate fear-irrelevant targets with more distractors, finding fear-relevant targets was independent of matrix size. Similar to the results from Experiment 2, participants decided more quickly that a target was not present with fear-relevant than with fear-imelevant matrices, and this effect was more evident with the small than with the large matrix.

More interesting, the effect of fear relevance was enhanced in fearful participants. Similar to controls, fearful participants were faster to find a fear-relevant target that they did not fear (e.g., a spider for a snake-fearful participant) than fear-irrelevant targets, but they were even faster to find a feared fear-relevant stimulus (e.g., a snake for a snake-fearful participant). It is a frequent clinical observation that phobic individuals tend to scan their environment for the feared stimulus. Spider phobics, for example, often examine a room they enter for spiders or signs of spiders, such as spider webs. Thus, the sensitivity to feared stimuli in fearful participants observed in this experiment illuminates one of the clinical characteristics of animal phobias. Similar results may be observed for other categories of phobia. For example, GilboaSchectman, Foa, and Amir (1999) reported that social phobics were faster than controls in detecting threatening angry faces in the visual search paradigm developed by Hansen and Hansen (1988). These findings fit into a voluminous literature showing an attentional bias for threat in anxiety patients (see, e.g., Mogg \& Bradley, 1998; and Williams, Watts, MacLeod, \& Mathews, 1997, for reviews). For example, Watts, McKenna, Sharrock, and Treize (1986) reported prolonged color-naming latencies for spiderrelated words in spider phobics, using the Stroop color-naming interference task. Subsequent studies have shown that a similar effect can be observed for masked word stimuli, suggesting that the locus of the effect is at an automatic level of information processing (van den Hout, Tenney, Huygens, \& de Jong, 1997). Similar data have been reported with Stroop paradigms adapted for use with pictures rather than words (Kind \& Brosschot, 1997; Lavy \& van den Hout, 1993).

Compared with interference paradigms, the present visual search procedure has several advantages when it comes to demonstrating attentional biases to threatening stimuli. First, it explicitly measures spatial attention, and from a functional perspective, the important task is to locate not only that there is a threat out there but in particular where it is located. Merely deciding that a threat is present may even be counterproductive, because without a notion where the threat is, a victim's attempts to escape actually can bring him closer rather than take him away from it. A functional attentional bias for threat, therefore, should be concerned

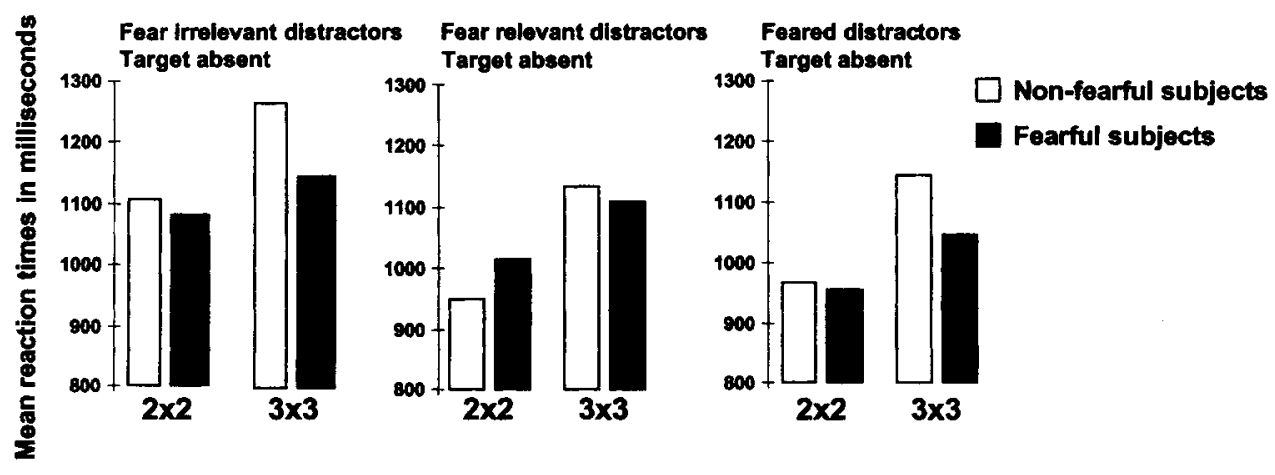

Figure 4. Mean reaction time for fearful and nonfearful participants to decide that a target was not present in matrices of fear-irrelevant, fear-relevant, and fear-relevant/feared distractors in Experiment 3. 
Table 3

Proportion of Errors for Matrices With and Without Targets in Experiment 3

\begin{tabular}{|c|c|c|c|c|c|c|c|c|}
\hline \multirow[b]{3}{*}{ Matrix size } & \multirow[b]{3}{*}{ Participants } & \multicolumn{4}{|c|}{ With targets } & \multirow{2}{*}{\multicolumn{3}{|c|}{ Without targets (distractors) }} \\
\hline & & & & & & & & \\
\hline & & FI dist. & FI dist. & Feared dist. & Nonfeared dist. & Feared & FR & FI \\
\hline \multirow[t]{2}{*}{$2 \times 2$} & High fear & .011 & .029 & .022 & .029 & .022 & .004 & .006 \\
\hline & Low fear & .018 & .018 & .007 & .004 & .022 & .011 & .017 \\
\hline \multirow[t]{2}{*}{$3 \times 3$} & High fear & .022 & .037 & .066 & .040 & .015 & .004 & .004 \\
\hline & Low fear & .026 & .033 & .033 & .022 & .007 & .000 & .007 \\
\hline
\end{tabular}

Note. "With targets" indicates the proportion of errors with fear-relevant (FR) and fear-irrelevant (FI) targets for small $(2 \times 2)$ and large $(3 \times 3)$ matrices and for participants high or low in fear of snakes or spiders. "Without targets" indicates the proportion of errors for feared (snakes for snake fearful), fear-relevant (spiders for snake fearful, snakes and spiders for low-fear participants) and fear-irrelevant distractors (dist.).

primarily with spatial attention. Second, a visual search paradigm provides a specific measure of the attention response. Note that in this experiment, the fastest responses of all were responses to the feared stimuli by fearful participants. In interference paradigms, on the other hand, the attentional bias is revealed in slowed responding, and responses may be slow for several reasons. For example, in the Stroop paradigm, the anxiety produced by emotional stimuli could directly interfere with the color-naming response rather than diverting attention to the content of the word. Thus, as a complement to dot-probe attention paradigms (e.g., MacLeod, Mathews, \& Tata, 1986), the visual search paradigm may prove useful in further elucidating the nature of attentional bias in anxiety disorder.

\section{General Discussion}

The results from the experiments reported in this article can be summarized in a number of broad generalizations. First, participants were consistently faster to find a fear-relevant stimulus (snake or spider) than a fear-irrelevant stimulus (flower or mushroom) against backgrounds of fear-irrelevant and fear-relevant stimuli, respectively. This finding suggest that humans share a predisposition to preferentially direct attention toward potentially threatening animal stimuli. Second, the latency of finding a fearrelevant target stimulus was independent of where in the matrix it was located. The latency to find a fear-irrelevant target, on the other hand, varied with its location such that detection was faster for locations close to the point of fixation. As a result, the advantage for the fear-relevant targets was evident particularly in locations requiring shifts of attention. Third, the latency of finding fear-relevant targets was not significantly increased when the number of distractors was increased from three to eight $(2 \times 2 \mathrm{vs.}$ $3 \times 3$ matrices). In contrast, the latency of locating fear-irrelevant targets was significantly prolonged by adding more distractors to the matrix. Fourth, deciding that no target was present in a matrix tended to be faster with fear-relevant than with fear-irrelevant distractors, particularly for small matrices. In no instance was the latency longer to decide about target absence with fear-relevant distractors. Thus, both for target present and target absent decisions, fear-relevant stimuli appeared to be processed more efficiently than the fear-irrelevant stimuli. Fifth, error rates were low, particularly for small matrices, and in general the participants were more accurate in locating fear-relevant than fear-irrelevant targets. Sixth, the bias for discovering threatening stimuli faster than nonthreatening ones was enhanced in participants specifically fearful of the target. Thus, snake-fearful participants were faster in locating snakes than spiders, even though they were faster with the spiders than with flowers or mushrooms. The opposite held for spider-fearful participants (i.e., their ordering of response latencies for target detection was spiders, snakes, and flowers or mushrooms. The fearful participants were overall less accurate than the nonfearful ones, particularly when deciding that there was a target among fear-relevant distractors, which suggests that fear produced by the distractors interfered with target detection.

This set of findings suggests that threatening stimuli were located in a preattentive, parallel-processing perceptual stage, whereas nonthreatening targets had to be searched for with a more laborious postattentive strategy. Thus, our conclusions are in substantial agreement with those for facial threat that Hansen and Hansen (1988) put forward in their classic article but subsequently retracted (Hansen \& Hansen, 1994). It is important to point out, therefore, that our study appears immune to the criticisms that have been taken as undermining the conclusions reached by Hansen and Hansen (1988). First, whereas the error rates they reported have been pointed out as suspiciously high (Byme \& Eysenck, 1995), our error rates were uniformly low, seldom exceeding $10 \%$. Second, findings that there were significant effects of location in the matrix for fear-relevant (angry faces) targets and that latencies for deciding about target absence were shorter for fear-irrelevant (happy faces) than fear-relevant (angry faces) distractors have been taken as evidence that both types of stimuli were serially searched for, and more efficiently so with fear-irrelevant stimuli (Hampton, Purcell, Bersine, Hansen, \& Hansen, 1989). In contrast, our results consistently failed to reveal an effect of target location for fear-relevant stimuli, whereas such an effect was repeatedly found with fear-irrelevant targets. Furthermore, target-absent responses were equal or faster in fear-relevant than in fear-irrelevant matrices. Finally, and perhaps most important, whereas Hansen and Hansen (1988) used only two fear-relevant and fear-irrelevant stimuli, which unfortunately were both confounded (Purcell et al., 1996), we used sets of fear-relevant and fear-irrelevant stimuli, which makes confounding by any irrelevant low-level physical factor highly unlikely. Not only must such a factor be common to snakes and spiders (and not shared by flowers and mushrooms), but according to Experiment 3 its effect must also be specifically enhanced for fearful participants. In particular, the observations that snake-fearful participants were faster in detecting snakes than 
spiders and that spider-fearful participants were faster in detecting spiders than snakes are very hard to reconcile with the notion of a low-level physical confounding factor behind the results. Rather, it appears more parsimonious to conclude that the critical factor was a high-level one: the threat value of the stimulus. This conclusion is further supported by the results of the experiments using schematic facial stimuli (Öhman et al., 2001). These stimuli used identical physical features to construct threatening and friendly faces, yet a discrepant threatening face was more quickly found than an equally discrepant happy face among neutral distractors. This convergence of results from the present series of experiments that used ecologically valid but less well controlled stimuli, and the previous series of experiments (Öhman et al., 2001) that used less ecologically valid but physically more well controlled stimuli, is reassuring. It strongly suggests that threat potential is an important factor in quickly capturing attention, as would be expected from the evolutionary perspective.

The efficiency of fear-relevant stimuli in capturing and perhaps holding attention could in fact contribute in two different ways to the faster location of fear-relevant than fear-irrelevant targets in the visual search paradigms we used. Because the search for fear-irrelevant targets occurred against a background of fearrelevant distractors, the distractors would tend to capture and hold attention to the expense of finding the target. However, if this were the case, one would have predicted longer latency in deciding the absence of a target in matrices of fear-relevant distractors than in those of fear-irrelevant distractors. The data, however, were quite the opposite: Shorter latencies existed for fear-relevant than for fear-irrelevant matrices without targets. Thus, we appear to be dealing with a genuine search asymmetry in which it is much easier to find snakes and spiders against background of flowers or mushrooms than the other way round.

In fact, this provides further support for the conclusion that the fear-relevant stimuli were located in a preattentive, parallel search, because search asymmetries have been taken as primary evidence for the operation of a preattentively processed elementary feature in the display (Treisman \& Gormican, 1988; Treisman \& Souther, 1985). For example, a circle with a small line perpendicular to its periphery is rapidly found among circle distractors with little effect of number of distractors, whereas finding a circle target among distractors consisting of circles with lines is heavily dependent on the number of distractors (Treisman \& Souther, 1985). The consistent search asymmetry that we observed suggests that our participants were inclined to regard fear-relevant stimuli as foreground and fear-irrelevant stimuli as background, or in a different terminology, they regarded the former as more interesting than the latter. Moreover, the search asymmetry implies that pictures of snakes and spiders include some elementary perceptual feature that makes them easy targets for the automatic capture of attention, whereas such features were not present in the pictures of flowers and mushrooms. This is in agreement with the claims made in the model presented by Öhman (1993) that postulated the existence of specific threat features that are preferentially picked up by an automatic significance evaluator and a system activating the autonomic nervous system. However, whereas the present results are in agreement with the existence of such elementary threat features, their nature still remains to be specified. For example, would they be related to the typical sinusoidal snake shape and to the circular body with protruding legs of the spider? Aronoff, Baraclay, and
Stevenson (1988) found that the latter shape, without any explicit reference to spiders or insects, was negatively evaluated in a rating study. This question may be more profitably pursued with facial stimuli that have more easily discriminable features. For example, Lundqvist, Esteves, and Öhman (1999) found that the V-shaped eyebrows of a schematic angry facial display were a powerful determinant for negative evaluation of faces. Furthermore, Öhman et al. (2001) showed that faces with such eyebrows were more rapidly and accurately located than faces with inverted $V$-shaped eyebrows (friendly faces) among both neutral and emotional distractors in a visual search task similar to that used in this study.

The results from Experiments 1 and 2 support the evolutionary hypothesis by demonstrating that humans in general appear to have a bias to attend to biologically relevant threatening stimuli in their surroundings. The stimuli we examined, snakes and spiders, are rated as moderately negatively valenced and arousing (e.g., Lang, Greenwald, Bradley, \& Hamm, 1993). Thus, they may be understood as moderately emotionally provocative, but probably more as interesting than as necessary to avoid, among normal participants in psychological experiments (Lang et al., 1993). For example, nonfearful participants looked more at spiders than at flowers when preference was assessed by eye movements (Hermans, Vansteenwegen, \& Eelen, 1999). Among the highly fearful participants of Experiment 3, however, they would be rated as very negative and arousing, and according to both voluntarily controlled viewing time (Hamm et al., 1997) and eye movements (Hermans et al., 1999), they would be avoided. Thus, for the participants selected to fear snakes or spiders in Experiment 3, one of the fear-relevant targets was considerably more emotionally provocative than the other. In agreement with the notion that emotion drives attention, the feared stimuli were more quickly picked up among distractor stimuli than any other targets (perhaps to allow quick avoidance). Thus, a stimulus factor, fear relevance, interacted with the emotional state of the individual to produce extremely efficient detection of feared targets (see Yantis, 1998).

Our data suggest that snake- and spider-fearful participants were sensitized specifically to have their attention captured by the feared stimulus. If emotions are understood as action sets (e.g., Frijda, 1986; Lang, 1984; Oatley \& Jenkins, 1996), which presume a set of goals, it could be suggested that emotion involves "attention control settings" (Folk et al., 1992) that make goal-relevant stimuli salient for the person. As a result, these stimuli may then automatically capture attention (Yantis, 1998). For example, avoiding and escaping from phobia-relevant stimuli are important goals for phobics, and achieving these goals is facilitated by automatically attending to phobia-relevant stimuli. Similarly, one would expect other emotional or motivational states to involve different attention control settings, for example, resulting in a bias for food-relevant items in the surroundings when one is hungry (see Mogg \& Bradley, 1998).

The fact that fear-relevant stimuli were found more quickly also among nonfearful participants suggests that there is a default attentional setting (see Folk et al., 1992), which is manifested as a general bias preferentially to direct attention toward evolutionarily fear-relevant stimuli among humans. This bias would reflect genuine preattentive automaticity of humans to orient toward animal threats. As such, it would be independent of prior conscious processing, whereas the additional bias shown by fearful participants may reflect postattentive automaticity that requires prior 
activation of fear (see Bargh, 1989). Similarly, the bias of highanxiety individuals to attend to threatening words appears to reflect postattentive automaticity (Fox, 1996). The general tendency, even among nonanxious individuals, to attend to threatening pictorial content in dot-probe paradigms (Mogg \& Bradley, 1999), on the other hand, may reflect the same type of generalized preattentive automaticity as displayed by the nonfearful participants in our experiments.

The present results may be taken as supporting the preparedness theory of phobias proposed by Seligman (1971) because they suggest that some common phobic stimuli have special psychological properties of likely evolutionary origin. However, the preparedness hypothesis specifically addresses the associability between potentially phobic stimuli and aversive outcome, in suggesting that such associations were easily formed, difficult to extinguish, and independent of cognition (Seligman, 1971). However, the procedure used in the present experiments did not address the associability of snakes and spiders with aversive outcome; aversiveness was addressed only indirectly in Experiment 3 because the fearful participants presumably were in an aversive emotional state when exposed to feared animals. Rather, these experiments were concerned with attention, or perceptual salience, that is, with properties of the stimuli themselves rather than with relationships between these stimuli and other events, even though the effects were modulated by an aversive emotional state. Indeed, in the original preparedness formulation, Seligman (1970) regarded perceptual salience as a confounding factor that had to be ruled out in experimental demonstrations of preparedness. For example, it was required that a potentially prepared stimulus was more effective than a nonprepared one only with aversive unconditioned stimuli for preparedness to be inferred. If the stimulus was more effective with any unconditioned stimulus, then its effectiveness could be attributed to intrinsic properties such as salience. Thus, the type of preferred attention that we have demonstrated for snakes and spiders in the present series of experiments may contribute to the likelihood that these stimuli become the object of phobias. After all, we are more likely to learn about stimuli that we attend to. Nevertheless, this is theoretically distinct from the enhanced associability with aversiveness implied by the preparedness hypothesis, and in fact, it could provide a potential confounding factor in demonstrations of preparedness.

A final word of caution may be appropriate in discussing the present results. This is because it has been debated in the human attention literature whether the visual search paradigm is a viable tool to distinguish between parallel and serial search. Wolfe (1998), for example, claimed that visual search data do not show a serial-parallel dichotomy supporting the notion of pop-out effects. Rather, he suggested that it is more sensible to talk about more or less efficient search, with efficient searches having a near zero slope of response latency on set size of searched features in the display, whereas very inefficient search would require $30 \mathrm{~ms}$ or more per searched item. Further caution is called for by the fact that the general visual search literature deals with very simple stimuli compared with the ones used in the present study. Thus, the basic stimulus features guiding search that have been isolated in this literature-color, orientation, curvature, motion, shape, and so forth-or conjunction of such features, are all very much simpler than the complex objects used as stimuli in the present series of studies. Accordingly, our search times were more than twice those typically reported in this literature. Thus, even though preattentive selection preferentially operates on objects (Duncan, 1984) and can operate on conceptual categories (Duncan \& Humphreys, 1989), searching for snakes, spiders, flowers, or mushrooms depicted in their natural surroundings appears a far cry from searching for a vertical line among slanted ones or a red dot among green ones (e.g., Wolfe, 1998). Given this vast difference in the perceptual complexities of the tasks, it is indeed surprising that finding fear-relevant stimuli among fear irrelevant ones so well conformed to the criteria for preattentive, parallel search suggested by Treisman (e.g., 1988). The appealing possibility, of course, is that the fear-relevant stimuli we have used contain a signature feature that, because of evolutionary history, is as readily picked up by the visual system as a vertical line among slanted ones. Yet, given the discrepancy in stimulus descriptions of the two contexts, one would like to see more concern for ecological validity among researchers on human attention and more concern for perceptual analysis among researchers in the fear and anxiety area. Thus, there is certainly need for much more research before researchers can conclude with any certainty that humans have perceptual and motivational systems that automatically pick up visual images of snakes and spiders to place them at the center of the attentional spotlight.

\section{References}

Aronoff, J., Baraclay, A. M., \& Stevenson, L. A. (1988). The recognition of threatening facial stimuli. Journal of Personality and Social Psychol. ogy, 54, 647-655.

Bargh, J. A. (1989). Conditional automaticity: Varieties of automatic influence in social perception and cognition. In J. S. Uleman \& J. A. Bargh (Eds.), Unintended thought (pp. 3-51). New York: Guilford Press.

Byrne, A., \& Eysenck, M. W. (1995). Trait anxiety, anxious mood, and threat detection. Cognition and Emotion, 9, 549-562.

Dimberg, U., \& Öhman, A. (1996). Behold the wrath: Psychophysiological responses to facial stimuli. Motivation and Emotion, 20, 149-182.

Duncan, J. (1984). Selective attention and the organization of visual information. Journal of Experimental Psychology: General, 113, 501517.

Duncan, J., \& Humphreys, G. W. (1989). Visual search and stimulus similarity. Psychological Review, 96, 433-458.

Esteves, F., Dimberg, U., \& Öhman, A. (1994). Automatically elicited fear: Conditioned skin conductance responses to masked facial expressions. Cognition and Emotion, 8, 393-413.

Folk, C. L., Remington, R. W., \& Johnston, J. C. (1992). Involuntary covert orienting is contingent on attentional control setting. Journal of Experimental Psychology: Human Perception and Performance, 18, $1030-1044$.

Fox, E. (1996). Selective processing of threatening words in anxiety: The role of awareness. Cognition and Emotion, 10, 449-480.

Frijda, N. (1986). The emotions. Cambridge, England: Cambridge University Press.

Gilboa-Schectman, E., Foa, E. B., \& Amir, N. (1999). Attentional biases for facial expression in social phobia. Cognition and Emotion, 13, 305-318.

Globisch, J., Hamm, A. O., Esteves, F., \& Öhman, A. (1999). Fear appears fast: Temporal course of startle potentiation in animal fearful subjects. Psychophysiology, 36, 66-75. 
Graham, F. K. (1992). Attention: The heartbeat, the blink, and the brain. In B. A. Campbell, H. Hayne, \& R. Richardson (Eds.), Attention and information processing in infants and adults (pp. 3-29). Hillsdale, NJ: Erlbaum.

Hamm, A. H., Globisch, J., Cuthbert, B. N., \& Vaitl, D. (1997). Fear and the startle reflex: Blink modulation and autonomic response patterns in animal and mutilations fearful subjects. Psychophysiology, 34, 97-107.

Hampton, C., Purcell, D. G., Bersine, L., Hansen, C. H., \& Hansen, R. D. (1989). Probing "pop-out": Another look at the face-in-the-crowd effect. Bulletin of the Psychonomic Society, 27, 563-566.

Hansen, C. H., \& Hansen, R. D. (1988). Finding the face in the crowd: An anger superiority effect. Journal of Personality and Social Psychology, 54, 917-924.

Hansen, C. H., \& Hansen, R. D. (1994). Automatic emotion: Attention and facial afference. In P. M. Niederthal \& S. Kitayama (Eds.), The heart's eye: Emotional influences in perception and attention (pp. 217-243). New York: Academic Press.

Hermans, D., Vansteenwegen, D., \& Eelen, P. (1999). Eye movement registration as a continuous index of attention deployment: Data from a group of spider anxious students. Cognition and Emotion, 13, 419-434.

James, W. (1890). The principles of psychology (Vol. 1). New York: Henry Holt \& Co.

Johnston, W. A., \& Dark, V. J. (1986). Selective attention. Annual Review of Psychology, 37, 43-75.

Jonides, J. (1981). Voluntary versus automatic control over the mind's eye's movement. In J. B. Long \& A. D. Baddeley (Eds.), Attention and performance IX (pp. 187-203). Hillsdale, NJ; Erlbaum.

Julesz, B., \& Bergen, J. R. (1983). Textons, the fundamental elements in preattentive vision and perception of textures. Bell System Technical Journal, 62, 1619-1645.

Kind, M., \& Brosschot, J. F. (1997). Phobia-related cognitive bias for pictorial and linguistic stimuli. Journal of Abnormal Psychology, 106, 644-648.

Klorman, R., Weerts, T. C., Hastings, J. E., Melamed, B. G., \& Lang, P. J. (1974). Psychometric descriptions of some specific fear questionnaires. Behavior Therapy, 5, 401-409.

Lang, P. J. (1984). Cognition in emotion: Concept and action. In C. E. Izard, J. Kagan, \& R. B. Zajonc (Eds.), Emotions, cognitions, and behavior (pp. 192-228). New York: Cambridge University Press.

Lang, P. J., Greenwald, M. K., Bradley, M. M., \& Hamm, A. O. (1993). Looking at pictures: Affective, facial, visceral, and behavioral reactions. Psychophysiology, 30, 261-273.

Lavy, E., \& van den Hout, M. (1993). Selective attention evidenced by pictorial and linguistic Stroop tasks. Behavior Therapy, 24, 645-657.

Lundqvist, D., Esteves, F., \& Öhman, A. (1999). The face of wrath: Critical features for conveying facial threat. Cognition and Emotion, 13, 691711.

MacLeod, C., Mathews, A., \& Tata, R. (1986). Attentional bias in emotional disorders. Journal of Abnormal Psychology, 95, 15-20.

Mogg, K., \& Bradley, B. P. (1998). A cognitive-motivational analysis of anxiety. Behaviour Research and Therapy, 36, 809-848.

Mogg, K., \& Bradley, B. P. (1999). Orienting of attention to threatening facial expressions presented under conditions of restricted awareness. Cognition and Emotion, 13, 713-740.

Müller, H. J., \& Rabbitt, P. M. A. (1989). Reflexive and voluntary orienting of visual attention: Time course of activation and resistance to interruption. Joumal of Experimental Psychology: Human Perception and Performance, 15, 315-330.

Oatley, K., \& Jenkins, J. M. (1996). Understanding emotions. Cambridge, MA: Blackwell.

Öhman, A. (1979). The orienting response, attention, and learning: An information processing perspective. In H. D. Kimmel, E. H. van Olst, \&
J. F. Orlebeke (Eds.), The orienting reflex in humans (pp. 443-472). Hillsdale, NJ: Erlbaum.

Öhman, A. (1993). Fear and anxiety as emotional phenomena: Clinical phenomenology, evolutionary perspectives, and information processing mechanisms. In M. Lewis \& J. M. Haviland (Eds.), Handbook of emotions (pp. 511-536). New York: Guilford Press.

Öhman, A. (2000). Fear and anxiety: Evolutionary, cognitive and clinical perspectives. In M. Lewis \& J. M. Haviland (Eds.), Handbook of emotions (2nd ed., pp. 573-593). New York: Guilford Press.

Ohman, A., \& Dimberg, U. (1984). An evolutionary perspective on human social behavior. In W. M. Waid (Ed.), Sociophysiology (pp. 47-86). New York: Springer-Verlag.

Öhman, A., Dimberg, U., \& Öst, L.-G. (1985). Animal and social phobias: Biological constraints on learned fear responses. In S. Reiss \& R. R. Bootzin (Eds.), Theoretical issues in behavior therapy (pp. 123-178). New York: Academic Press.

Ohman, A., Lundqvist, D., \& Esteves, F. (2001). The face in the crowd revisited: A threat advantage with schematic stimuli. Journal of Personality and Social Psychology, 80, 381-396.

Ǒhman, A., \& Mineka, S. (2001). Fears, phobias, and preparedness: Toward an evolved module of fear and fear learning. Psychological Review, 108, 483-522.

Parra, C., Esteves, F., Flykt, A., \& Öhman, A. (1997). Pavlovian conditioning to social stimuli: Backward masking and the dissociation of implicit and explicit cognitive processes. European Psychologist, 2, 106-117.

Pashler, H. (1987). Detecting conjunctions of color and form: Reassessing the serial search hypothesis. Perception and Psychophysics, 41, 191201.

Posner, M. I. (1978). Chronometric explorations of mind. Hillsdale, NJ: Erlbaum.

Posner, M. I. (1980). Orienting of attention. Quarterly Joumal of Experimental Psychology, 32, 3-25.

Posner, M. I., \& Petersen, S. E. (1990). The attention system of the human brain. Annual Review of Neuroscience, 13, 25-42.

Purcell, D. G., Stewart, A. L., \& Skov, R. B. (1996). It takes a confounded face to pop out of a crowd. Perception, 25, 1091-1108.

Ratcliff, R. (1993). Methods for dealing with reaction time outliers. Psychological Bulletin, 114, 510-532.

Schneider, W. (1988). Micro Experimental Laboratory: An integrated system for IBM PC compatibles. Behavior Research Methods, Instruments \& Computers, 20, 206-217.

Schneider, W., Dumais, S. T., \& Shiffrin, R. M. (1984). Automatic and control processing in attention. In R. Parasuraman \& D. R. Davies (Eds.), Varieties of attention (pp. 1-27). Orlando, FL: Academic Press. Seligman, M. E. P. (1970). On the generality of the laws of learning. Psychological Review, 77, 406-418.

Seligman, M. E. P. (1971). Phobias and preparedness. Behavior Therapy, 2, 307-320.

Sokolov, E. N. (1963). Perception and the conditioned reflex. Oxford, England: Pergamon Press.

Thorpe, S. J., \& Salkovskis, P. M. (1995). Phobic beliefs: Do cognitive factors play a role in specific phobia? Behaviour Research and Therapy, 33, 805-816.

Tooby, J., \& Cosmides, L. (1990). The past explains the present: Emotional adaptations and the structure of ancestral environment. Ethology and Sociobiology, 11, 375-424.

Treisman, A. (1988). Features and objects: The Fourteenth Bartlett Memoxial Lecture. Quarterly Journal of Experimental Psychology, 40A, 201-237.

Treisman, A., \& Gelade, G. (1980). A feature integration theory of attention. Cognitive Psychology, 12, 97-136.

Treisman, A., \& Gormican, S. (1988). Feature analysis in early vision: Evidence from search asymmetries. Psychological Review, 95, 15-48. 
Treisman, A., \& Souther, J. (1985). Search asymmetry: A diagnostic for preattentive processing of separable features. Joumal of Experimental Psychology: General, 114, 285-310.

van den Hout, M. A., Tenney, N., Huygens, K., \& de Jong, P. J. (1997). Preconscious processing bias in specific phobia. Behaviour Research and Therapy, 35, 29-34.

Watts, F. N., McKenna, F. P., Sharrock, R., \& Treize, L. (1986). Colour naming of phobia-related words. British Journal of Psychology, 77, 97-108.

Williams, J. M. G., Watts, F. N., MacLeod, C., \& Mathews, A. (1997). Cognitive psychology and the emotional disorders (2nd ed.). New York: Wiley.
Wolfe, J. M. (1998). Visual search. In H. Pashler (Ed.), Attention (pp. 13-73). Hove, England: Psychology Press.

Yantis, S. (1998). Control of visual attention. In H. Pashler (Ed.), Attention (pp. 223-256). Hove, England: Psychology Press.

Yantis, S., \& Johnson, D. N. (1990). Mechanisms of attentional priority. Journal of Experimental Psychology: Human Perception and Performance, 16, 812-825.

Received September 30, 1999

Revision received August 1, 2000

Accepted August 1, 2000

\section{New Editors Appointed, 2003-2008}

The Publications and Communications Board of the American Psychological Association announces the appointment of five new editors for 6-year terms beginning in 2003.

As of January 1, 2002, manuscripts should be directed as follows:

- For the Journal of Applied Psychology, submit manuscripts to Sheldon Zedeck, PhD, Department of Psychology, University of California, Berkeley, CA 947201650.

- For the Journal of Educational Psychology, submit manuscripts to Karen R. Harris, EdD, Department of Special Education, Benjamin Building, University of Maryland, College Park, MD 20742.

- For the Journal of Consulting and Clinical Psychology, submit manuscripts to Lizette Peterson, PhD, Department of Psychological Sciences, 210 McAlester Hall, University of Missouri-Columbia, Columbia, MO 65211.

- For the Journal of Personality and Social Psychology: Interpersonal Relations and Group Processes, submit manuscripts to John F. Dovidio, PhD, Department of Psychology, Colgate University, Hamilton, NY 13346.

- For Psychological Bulletin, submit manuscripts to Harris M. Cooper, PhD, Department of Psychological Sciences, 210 McAlester Hall, University of Missouri-Columbia, Columbia, MO 65211.

Manuscript submission patterns make the precise date of completion of the 2002 volumes uncertain. Current editors, Kevin R. Murphy, $\mathrm{PhD}$, Michael Pressley, $\mathrm{PhD}$, Philip C. Kendall, PhD, Chester A. Insko, PhD, and Nancy Eisenberg, PhD, respectively, will receive and consider manuscripts through December 31, 2001. Should 2002 volumes be completed before that date, manuscripts will be redirected to the new editors for consideration in 2003 volumes. 